\title{
USING ONTOLOGIES FOR THE EMERGENCY RESPONS SUPPORT: A SURVEY
}

FASEE ULLAH ${ }^{1}$, AND IMRAN KHAN ${ }^{2}$
'Department of Computer Science, Abasyn University, Pakistan
fasee.ullah@ abasyn.edu.pk
2Department of Computer Science, Abasyn University, Pakistan
Imran.khan@ abasyn.edu.pk
Received June 2015
ABSTRACT. Ontology is an efficient and an adequate way of conceptualization of
knowledge representation for real world applications. Ontologies are successfully
used in varieties of knowledge domains. In this paper, we focus on the field of
medical ontology knowledge construction and representation. We identified three
different medical ontologies classes: Generic, Specific and Mass Casualties
Incidents (MCI) medical ontologies. This survey should support the construction of
medical Ontologies in future for a better patient treatment. In particular, we survey
and focus on the existing medical related ontologies and investigate their suitability
for use in Mass Casualties Incidents (MCI).

Keywords: Mass Casualties Incidents (MCIs); Medical Ontology; Generic ontology; Specific ontology.

1. Introduction. The World Health Organization (WHO) has published updated report on death in June 2011 due to various disorders and disasters. This death ratio is reported in millions [1]. Due to this, WHO has categorized countries on income based with the following death rates information [1] as follows.

1. Low-income Countries death rate in millions ranges from 0.21 to 1.05 .

2. Middle-income Countries death rate in millions ranges from 0.83 to 5.27.

3. High-income Countries death rate in millions ranges from 0.17 to 1.42 .

4. World based death rate in millions ranges from 1.21 to 7.25 .

These death rates are in millions include all types of death rate from normal disease death range to high level serious disease death range.

Emergency response system is the important system to save the lives of the victim people on time from disasters such as Earthquakes, Plane crash, Flood, Explosion, Wild re, underground construction, Car incidents, Cave or building collapses, etc [45]. After 9/11 incidents in the United States, all the existing security protocols are strengthen and new security protocols are developed to handle the worst case situations [46]. A lot of researches have been done and still are on going to introduce new mechanisms to save the humanity from disasters. Due to this, a manual emergency response support system is introduced, that is Mass Casualties Incidents (MCI). MCI is one of them newly proposed security protocol to measure and handle all kinds of situation during at MCI locations. For this purpose, the newly manual system is introduced in three phases to help injured people which are Triage, Treatment and Transportation system [45][46][47][50]. However, this manual system also takes a lot of time to recognise the injured people level and provide proper treatment to them. Due to this long process, the death cases are increased of unavailability of automatic and intelligent system to calculate the seriousness level of the injured people and provide treatment resources on time with some predefined medical instructions.

In the current situations, we need a specific and intelligent systems which monitor health continuously or for 
some specific period of time (hajj and umrah) [2] with some automation. For this purpose, various existing research tries to design and develop a concept is known as ontology. Ontologies significantly support automatic calculation on the basis of predefined knowledge with some intelligent classes and properties denote and perform specific actions on disorders.

There are three medical ontologies presented in this work such as: Generic medical ontology, Specific medical ontology and Mass Casualties Incidents (MCI) based medical ontology. This MCI focuses on Health-oriented ontologies, and Operation support ontologies to provide treatment facilities to victim people on time of any kind of disaster by using communication infra structure to give detail information of the shortest routes to the hospital, number of available beds in hospitals and list of treatment facilities in the hospital etc are the core benefit of the Mass Casualties Incidents (MCIs) ontologies.

In a generic medical ontology, we have combined the maximum number of existing general purpose medical ontologies which show the requirements how to design the general purpose medical ontology to treat the patient via internet.

In a specific medical ontology, we have also covered maximum number of medical ontologies related to the specific patient disorders issue such as breast cancer, mood disorder and strokes etc are discussed with their designing rules.

The Mass Casualties Incidents (MCI) is a new term used in medical ontology to calculate resources automatically (medical staff, air ambulance, hospitals, shortest routes etc) and provide treatment to the victim people of the accident location on time is considered for future health treatment purposes. Although this Mass Casualties Incident (MCI) system is operated manually, like as START system [45]. There are many types of MCI design can be available for health and safety such as MCI general, MCI Health, MCI operation based and MCI hybrid etc. So, we consider mainly two MCI's, MCI Operation support ontologies and MCI Health-Oriented ontologies because these two MCI's cover all types of MCI ontologies.

The purpose of these types of MCI medical ontologies can use for the clinical guidelines for the physicians, specialists, patients and academics to create views of medical ontologies [3] to save life of humanity. The advantage of this paper is to combine all the existing medical ontologies data and analyse how to relate these data for MCI victim people which can help better in the designing of emergency response support system. The reminder of the paper is constructed as follows: Fundamentals of the ontologies contain in Section 2. Classification of the ontologies are presented in Section 3. Section 4 contains Survey of the Existing Health Ontologies. Section 5 contains Critical Review and Future Research Directions. Final Section 6 is a conclusion.

2. Fundamental of the Ontologies. The ontology concept come from philosophy and used by Artificial Intelligence (AI) community as an explicit specification of conceptualization [8]. Ontology provides various forms to represent knowledge such as taxonomy, vocabulary lists and thesaurus [12] concept based on the collection of domain specific knowledge. Ontology design and construction varies from field to field that represents the knowledge in the form of formal language description. However, ontology expresses the knowledge very efficiently for medical science fields. Therefore, generally most of the modes define two types of ontologies which are known as domain ontology and theory ontology [4]. The domain ontology always deals with detail description of classes and subclasses, relationships between classes (subclasses), properties and instances (object) while theory ontology explains the concrete directions of the area or domain.

Representation of domain knowledge is mostly represented in a single ontology, multiple ontology, and hybrid ontology [5] while construction is the iterative process which refines and improves the knowledge structure by domain experts with five step processes [6] as follows.

Design: This defines the scope of the domain and relationships between classes.

Develop: various steps involve to construct an ontology from existing ontologies or defines a new specification and also requires Top Down and Bottom Up approach.

Integrate: to fulfill the requirement of the ontology, can combine existing ontologies.

Validate and Feedback: After successful completion of the ontology can verify by domain experts with 
professional tools.

Iterate: Modify or update the developed ontology with the suggestion of the domain experts.

The manual, semi automatic and full automatic steps [6] can involve in the analysis and development of ontology.

A conceptualization is an abstract that contains domain specific knowledge with vocabularies in the form of subjects and objects. The authors of [9] have defined that the ontology is the science and "structure of objects, properties, events, processes and relations in every area of reality ..." [9]. The authors of [8] have defined some basic rules apply on ontology for knowledge management such as knowledge creation and, knowledge store. This store knowledge must be evaluated or matched when requires. Ontology creates a tree like structure and shares knowledge with the communities or designer of ontologies in a specific order to execute knowledge in the proper way. The structure of adopted ontology consists of categories, relationships, attribute, constraints and instances with the following steps to construct medical ontology as follows [10].

1. Follow the contents of medical sciences and create a medical ontology framework (domain).

2. Draw the important concepts to present a real time system.

3. Make relationships between concepts (e.g. Properties, attributes etc).

4. After completion of the required information for the construction of medical ontology, use an appropriate tool (e.g. protégé 2000) to develop the required ontology (in this case is only medical ontology).

5. Test and validate the system is with the help of field experts.

Ontology construction of knowledge domain varies due to knowledge repository of maximum sources (dictionaries, social websites etc) and also varies from community-to-community knowledge sharing. These knowledge repositories confuse learner of any domain due to same word having multiple meanings such as "corpus, database, repository, storage, dictionary, and ontology ...[11]". Therefore the authors of paper [11] have presented a academic domain ontology which facilitate both human and machine agents to store, systematize and manage the complex knowledge with three main objectives regarding knowledge repository as shown below.

1. The knowledge repository should not be limited to support essential features of academic knowledge.

2. The gathered knowledge could help in the construction of knowledge repository. However, the best practices in this construction is a human to support in good directions.

3. After combining knowledge repositories must be shared among communities.

Knowledge sharing of heterogeneous ontologies is another issue faced during design of the medical ontology. Therefore, three major types of heterogeneous ontologies are discussed. Those are mapping, merging and integration [10]. The mapping ontology helps to combine various types of heterogeneous ontologies knowledge with common relationships. These heterogeneous ontologies such as $\mathrm{O}_{1}, \mathrm{O}_{2}$ etc are combined with a common shared ontology. While one-to-one mapping is the second type of heterogeneous ontology mapping. This type of mapping is created with support of direct and indirect paths of ontologies to share knowledge with each other and integration helps to combine various domain knowledge sources.

An intelligent and efficient ontology development requires some basic steps. However, these steps vary from application to application such as medical ontology and physiological ontology. Medical ontology is needed four basic steps to create a medical domain knowledge for disorders which are:

1)types of various diseases or injuries

2) description of each disease

3)causes to occur a specific disease

4) precautions and treatment procedures.

Other definitions of ontology are concepts, thesaurus and taxonomy [12] terms also used to represent knowledge organization. Thesaurus comprised of terms and these terms are the basic vocabulary to define and control language indexes and relationships between them while taxonomy is indexing language which keeps knowledge an arranged form of different domains and assigns alphanumeric letters to each knowledge.

[7] Follows different steps towards successful analysis, design and implementation of the medical ontology as follows.

Analysis of the domain: Identification of the problem is the first step towards successful designing of the medical ontology as we learnt from software development processes. Although in medical sciences, domain 
experts are needed to achieve the targeted answers and designing that helps in production of medical ontology.

Conceptualization of the domain knowledge: All the physical processes can find from medical centre environment such as a patient registration, OPD, wards,medical tests, and reports etc.

Implementation, refinement and evaluation: This process is also called iterative process which periodically checks the underlying software to match the requirements of the medical ontology and patient treatment requirements.

3. Classification of the Medical Ontologies: Medical ontology is providing various methods for construction of medical treatment domains for the patients and these construction varies from source to source and problem to problem. We combined three basic methods for medical ontologies which will help in future in the construction, and making priority classes of each disorders. To achieve these objective, this survey paper presents three kinds of medical ontologies which are: GenericMedical Ontologies, Specific Medical Ontologies and MCI based Medical Ontologies are shown in Fig 1 and each ontology has further sub classes to show what kind of disorders discussed or steps provided to help in construction of a generic or a specific medical treatment ontology. The construction of such medical ontologies need a dedicated professional team to clarify all the things related to the domain problem and also is a challenging task in the medical ontology field. Due to urgency based issues, these three Medical Ontologies are explained one by one respectively.

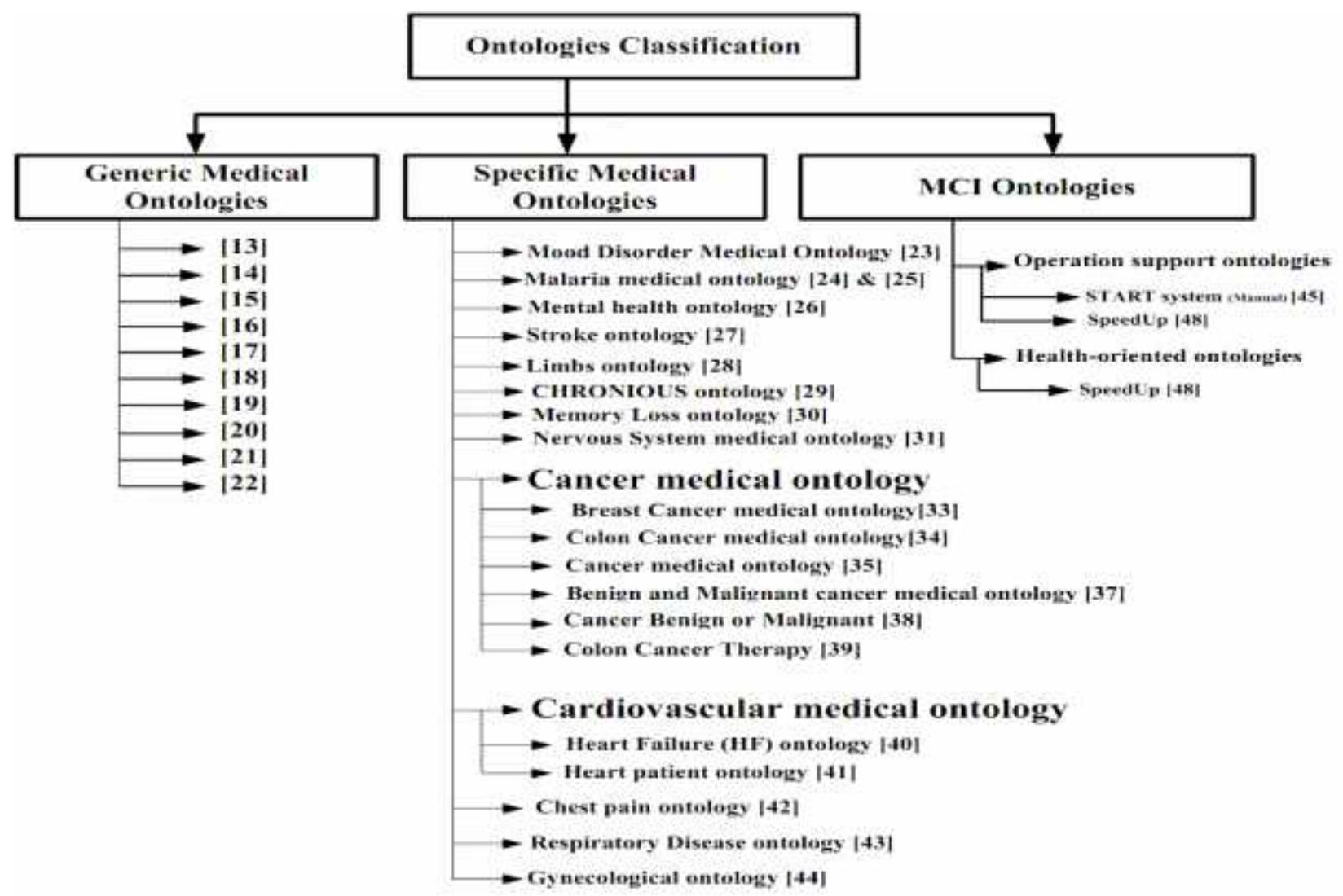

Fig 1. Classification of Medical Ontologies

In general medical ontologies, Generic Human Disease ontology [13] has developed with four basic properties to nd the disease of the patient as follows.

\footnotetext{
4 Disease type

Disease symptoms

Relevant causes to the disease

Number of available treatment for a disorder
} 
Agents are used to resolve conicts of the same word having more than one definitions such as gene is used [14]. Agents work on certain rules with the association of Decision support system and Artificial Intelligent knowledge base. [15] provided instructions to remove conflicts having more than one meaning of the one word with the following conditions to achieve patient diagnoses successfully.

- First of all, search, understand, and construct the relevant queries are required regarding the availability of information resources.

- To retrieve the appropriate data from various information resources and merge those information with different information resources when same word has more than one definitions.

Healthcare Context Information model [16] is developed to provide health services to the patient everywhere with advanced communication infrastructure. This model is needed a patient information to treat her on time with the following data.

- Individual data: Name, Age, history of medicine used etc.

- Medicine data: Disease data, symptom data, Examination data, and health data.

- Service data: bio data analysis and so on more ...

Except these information, other data are also used to evaluate the patient disorder for better health care.

Heart Failure patient medical ontology has designed with the help of Decision Support System (DSS) [17]. This DSS system has connected with ontologies, DSS controller, DataBase, and reasoner etc to conclude the factual information of the heart patient. The biometric device is used to obtain the patient history data.

This article [18] presents three phases in the construction of the medical domain ontology. In these phases, 1) Collect relevant information from various sources via internet, 2) Domain Modelization and 3)Meta conceptualization presents relationship between local and global knowledge sources.

Chronic Disease Management [20] ontology is designed with the help of Electronic Medical Record (EMR) and other related clinical vocabularies which were supported by HL7 RIM and Computer based Patient Record (CPR)[20]. Ontologies and Agents based BioSTORM [21] has developed with following steps.

i. Create domain ontologies

ii. Create domain methods

iii. Create conguration knowledge bases

iv. Generate JADE interfaces from the model

v. Deploy and execute

The Clinical Practice Guideline of Diabetic Retinopathy (GLDR) [22] has developed with instructions of medical professional team. During designing of medical ontology system, a dedicated team helped them in the identification of the problems and to convert them in semi-automatic way for better patient health care.

The section gives an overview of the specific medical ontologies for disorder patients.

\section{Mood Disorder Medical Ontology}

Haghighi-Koeda (HK) [23] medical ontology has developed for a mood disorder patient with the support of medical and psychological ontologies patterns to treat patient on time through web access.

\section{Malaria medical ontology}

The seven partial parameters have used for malaria patient to construct a medical ontology prototype, is known Human Malaria Control Ontology (HMCO) [24]. The seven partial parameters are: (1) Malaria vectors (2) Malaria types, (3) Malaria parasites, (4) Malaria Symp- toms, (5) Malaria treatment (prevention, therapy), (6) Epidemiology data on malaria, and (7) Malaria Control [25].

\section{Mental health ontology}

Mental Health ontology [26] has proposed with three main parent classes such as disorder types, factor and treatment are further connected with sub classes. 


\section{Stroke ontology}

Hemiparesis is the common disease to damage some part of the body such as leg, and arm etc after stroke. The NeuMORE [27] is the well known a specific medical ontology, has defined 4 phases for construction of Hemiparesis patient disorder ontology.

\section{Limbs ontology}

This OSMMI [28] ontology has attempted to achieve goals of four problems related to the lower limbs.

\section{CHRONIOUS ontology}

CHRONIOUS [29] system has introduced to monitor the Chronic Obstructive Pulmonary Disease (COPD) and Chronic Kidney Disease (CKD)with support of wearable T-shirt sensing infrastructure to monitor patient periodically and inform doctors for treatment.

\section{Memory Loss ontology}

This memory loss framework is known as Aloa-aidants.fr [30] and has developed with support of social semiotic ontology and Aloa OIR (Open Information Retrieval) system based. The proposed framework contains three views to analyse the queries of the social communities and makes the Aloa OIR system with expert teams to suggest treatment for memory loss patient.

\section{Nervous System medical ontology}

Neurological Disease (ND) ontology and NeuroPsychological Testing Ontology (NPT) [31] are considered as medical ontologies for Nervous system of the human body. The primary concern of ND ontology is on Alzheimer disease which has memory loss related diseases often occurs in 40 and 50 year ages. Both ND and NPT ontologies have developed with support of OBO Foundary [32] and with some other external ontologies. ND ontology contains 400 classes, 250 textual definitions and more than 150 classes have logical definitions while in NPT ontology contains 250 specific classes.

\section{Cancer medical ontology}

\section{Breast Cancer medical ontology}

The Breast Cancer (BC) ontology model has designed with support of Clinical based Decision Support System [33]. Eight classes have defined for BC disorder such as Patient Type, Physician Type, Illness, Menstrual Status, Recommendation, Symptom, Diagnostic Tests, Treatment, Age, Risk Factor, Weight Status and Patient Wish with other supportive disjoined classes (Recommendation and Diagnostic Tests, and Recommendation and Treatment.) for treatment aspects [33].

\section{Colon Cancer medical ontology}

The authors of OWL ontology will create the ontology of MATCH CC (Colon Cancer) [34] from existing ontologies to know the current state of the patient. The MATCH architecture occurs in two parts. In the first part, CC ontology has created with support of the patient profile, is known as discovery phase while in second part, all the discovered information have mapped in relation database schemas depicted.

\section{Cancer medical ontology}

The clinical and molecular data are combined with the help of Basic Formal Ontology (BFO) [36] steps to design cancer ontology from existing available ontologies such as the National Cancer Institute Thesaurus (NCIT), Gene Ontology (GO) and Foundational Model of Anatomy (FMA). The ACGT MO (Advanced Clinico-Genomic Trials Medical Ontology) [35] ontology has developed to treat the patient by using Ontology-based Trial Management System (ObTiMA) [35].

\section{Breast Cancer: Benign and Malignant cancer medical ontology}

The Wisconsin Breast Cancer Dataset (WBC) [37] ontology has used as a case study to create a new breast cancer ontology engine that contains two phases. In phase 1, this algorithm matches the searched knowledge and prepares the rules whether searched knowledge is available in the datasets or not. Phase 2 contains ontology development algorithm engine rules and these rules further define concepts. This concept describes a specific domain with assumption and domain properties. In this algorithm, it contains 25 programming rules 
as IF THEN ELSE, FOR, DEFINE, MAPPING, END, VARIOUS MATCHING RULES, EQUAL, NOT EQUAL etc. This algorithm is used for Benign and Malignant cancer concepts with attribute values from 1 to 10 which define ranges from normal to abnormal situations of a cancer patient.

\section{Cancer Benign or Malignant}

Case-Based Reasoning (CBR) [38] ontology classifier has used with two open source frameworks jCOLIBRI and myCBR to find whether the patient involves in the Benign cancer or in Malignant. The jCOLIBRI is available in two versions. The version 1 myCBR is selected for designing and version 2 is related to the developer. The jCOLIBRI is based on some reasoning structure cases while myCBR is based on classes and properties and easily implemented on protégé tool. The myCBR is a best tool for developing of a new CBR based application for breast cancer classifier [38].

\section{Colon Cancer Therapy}

The proposed colon cancer therapy ontology has developed and known as Disease-treatment ontology [39]. The proposed ontology attempted to include five classes such as: Disease, Treatment, Condition, Effect and Evidence. Further, these classes have defined subclasses with different relationships.

\section{Cardiovascular ontology}

\section{Heart Failure (HF) ontology}

This paper has proposed Heart Failure (HF) ontology [40] and divided heart ontology into five main super classes which are: HF Concept, Patient characterises, Testing, Treatment and Patient [40]. Each parent class has further connected with sub classes except patient class has no sub class defined. In HF concept ontology included heart failure abnormal issues, medical synonyms and other types etc. The patient characteristic includes possible signs, diagnoses and symptoms etc. Tests included physical tests of the body while Treatment included medications, devices or other recommendation related to the HF.

\section{Heart patient ontology}

The Synthetic measurement system has proposed and comprised main sources to remotely monitor the heart patient with decision support system such as clinical DSS (HIMS) data, ontology, Sensors, Patient EHR analysis, existing literature, guideline etc [41]. These sources of information are connected with patient mobile and clinician mobile to get data from patient wearable sensors.

\section{Chest pain ontology}

The proposed chest pain ontology framework is based on the dynamic questionnaires. This framework contains four components with two GUIs (one GUI is for patient and other for doctors to recommend further steps against chest pain) which are adaptive questionnaire, patient and family medical history, risk assessment and clinical decision support system [42]. These components have connected with various chest pain classifications guidelines units such as NICE, ACC (American College of Cardiology) and SIGN (Scottish Intercollegiate Guideline Network) to evaluate chest pain disorder patients.

\section{Respiratory Disease ontology}

The respiratory system ontology [43] has developed with the following classes and subclasses. The parent root node class is Respiratory diseases with connected sub classes: pneumoconiosis and other lung diseases due to external agents, Acute Respiratory infection, chronic obstructive pulmonary disease and allied conditions etc are the sub classes of the parent node class and further these sub classes have connected with other sub classes to return query results.

\section{Gynecological ontology}

The proposed ontology architecture has ontology-Based Psychiatric Disorder Detection System and focused on the stress because stress is one of the leading disorder approach towards life threatening issues such as heart attack and cancer [44]. So proposed ontology has divided into two types: ontology task and ontology domain, in which ontology task will be mapped on ontology domain to satisfy the required results. Further, 
this architecture contains GUI, inference mechanism, ontology domain, ontology task, decision tree algorithm and Maridian with DSM (Diagnostic and Statistical Manual of Mental Disorders) [44].

\section{Mass Casualties Incidents based Medical ontology}

Mass Casualties Incidents (MCI) can occur anywhere. There are many systems exist to help the victim people, however all these systems are operated manually with human support. Lots of human resources and Technology are needed to help the victim people at the incident locations. So the current situation demands to replace the existing systems with automatic systems. For this purpose, operation support ontologies and Health-oriented ontologies are defined in the MCI as shown in Fig 1. The operation support ontologies focus to provide communi- cation infra structure as explained in section $4 \mathrm{C} 1$. A relevant underlying example is SpeedUp [48] project which focuses on SpeedUp practice and SpeedUp Technology. In the Health oriented ontologies, START System [45] has introduced to provide treatment facilities to the victim people of mass casualties incident locations but this START system is operated manually with support of medical staff and other helping staff members. However, existing infra structure needs more work to build up a perfect system for victim people at the mass causalities incident locations.

In existing survey of medical ontologies, This paper suggests some partial steps to make an MCI ontology as follows.

Rule 1. The papers $[13,15,16,17]$ have defined some rules for generic medical ontologies. These rules can help to design a specific medical ontology for a specific disease.

Rule 2. The specific medical ontologies have discussed with given specific disease ontologies in this paper.

Rule 3. If rule 1 apply on the rule 2 with deep study and refinement aspects, can achieve a problem narrow solution.

Rule 4. Write an algorithm for rule 3 and implement in sensor devices. These sensor devices must deploy on the human body to monitor continuously for any specific disorder issue. Moreover, these sensors connect with smartphones to inform medical sta

for urgency

case and provide a particular treatment to the victim.

These rules can make an efficient and alert system for 24/7/365 times to monitor and protect humanity for any loss and damage.

\section{Survey of the Existing Health Ontologies}

From treatment aspects, ontology based medical domain construction and implementation for victim people of various injuries as well as for patients in different disorders is one of the automatic leader technology to recognize the injuries levels and the patient disorders during Mass Casualties Incidents (MCI).

This paper covers the maximum available existing related work to the generic medical ontologies, specific medical ontologies and MCI ontologies in subsections.

A. Generic Medical Ontologies

CHD (Coronary Heart Disease) Medical ontology has created from existing the Chinese Traditional Medical and Western Medical structure [10] databases. Due to complex and scattered heterogeneous resources of medical ontologies, Generic Human Disease ontology [13] has developed with the following four basic properties and also shown in Fig 2 [13].

Disease type

Disease symptoms

Relevant causes to the disease

Number of available treatment for disorder 


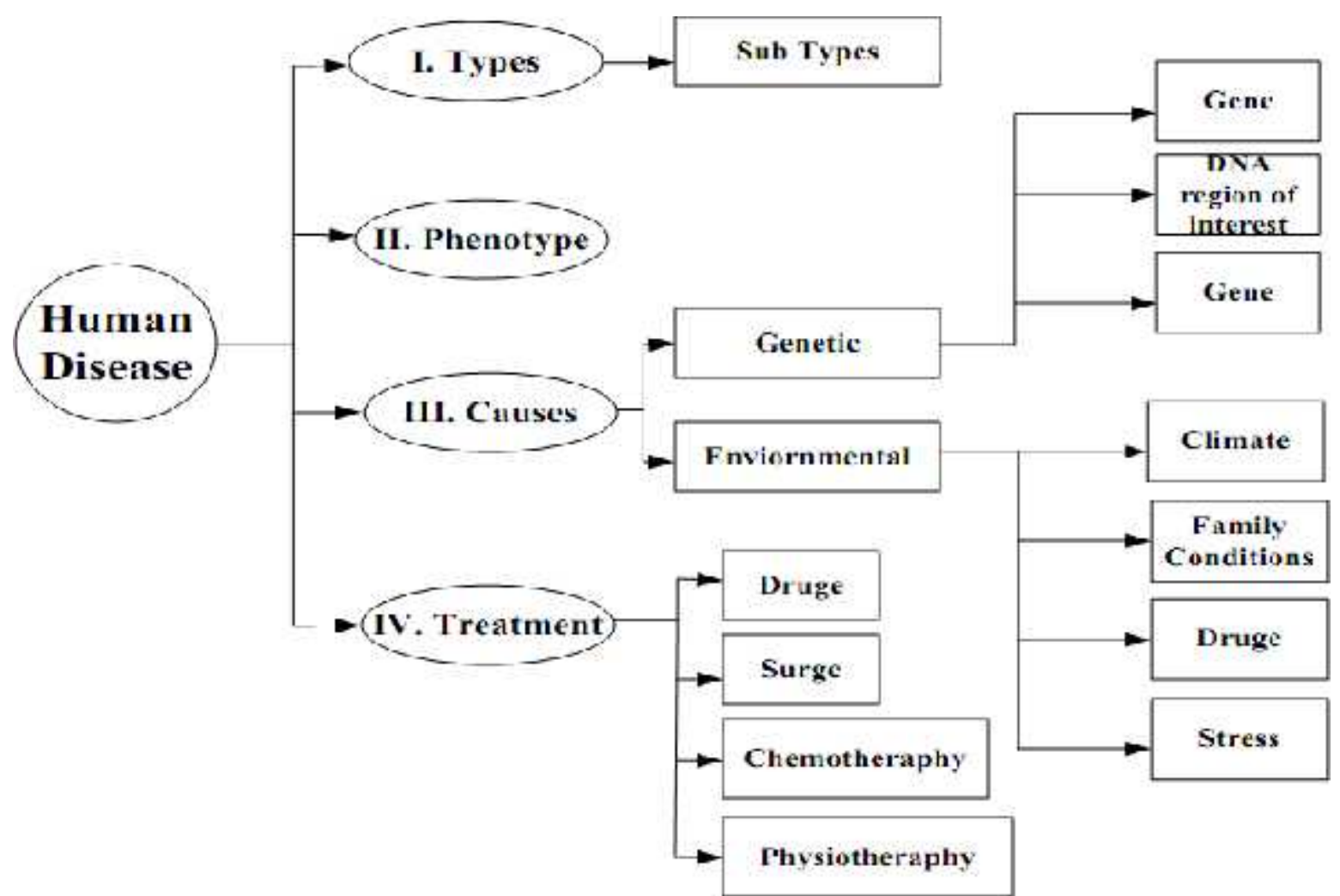

Fig. 2. Generic Human Disease ontology and its four main subontologies: Types, phenotype, causes and treatment [13]

This paper [14] focuses on agents and ontologies how to differentiate between generic and specific human disease ontologies because biomedical information resources are distributed all over the global village. Due to these rapid increases in the information resources on internet, it has created complicated problems when browsing and searching specific items. Some important instructions presented in [15] when searching specific items as follows:

First of all, search, understand, and construct the relevant queries are required regarding the availability of information resources.

4 To retrieve the appropriate data from various information resources and merge those information with different information resources when same word has more than one definitions.

The availability of more than one information resources on the same topic can create complexity when data about a particular search is available but cannot compatible with each other due to the way information is structured and organized in databases. Due to this complexity, cannot automatically compare and analyse them such as Gene [14] shown below with more than one definitions.

Gene $=$ A part of DNA sequence

Gene $=$ is a composed of $\mathrm{A}, \mathrm{C}, \mathrm{G}$ and $\mathrm{T}$ nucleotides

Gene $=$ Codes for a protein

Gene $=$ is situated on a chromosome

These kind of issues can be resolved with the help of ontology agents. In a first case, when same keyword is available (e.g. Gene) with different definitions in databases, agents can resolve this conflict of various definitions in databases with certain rules. While in second case, different databases (ontologies) have different agents with divverent definitions of the same word. So these types ontologies can then be merged into a large ontology for agents to remove conflicts. 
The Healthcare Context Information model has developed and is based on medical ontology in order to provide services in ubiquitous environments [16]. This development is performed due to less scalability and device dependability issues in existing conventional U-healthcare services system. The authors have also focused on the communication and sensing technologies which is a kind of lack and less communication with injured persons.

The table I [16] shows objectives how to combine various services (e.g. patient data, medical data, location data etc) to make the healthcare context information system efficient. These services are related to the human body to measure the health status of individual patient by using laptops, PCs and smartphones connect to health services system.

\begin{tabular}{|l|l|}
\hline Types of Data and Services & Attributes and Symptoms \\
\hline Individual data & $\begin{array}{l}\text { Name, age, ex, injection history, } \\
\text { disease, disease history, family } \\
\text { disease history, grade, job, income, lifestyle, smoke, } \\
\text { drink }\end{array}$ \\
\hline Medicine Data & $\begin{array}{l}\text { Disease Data Hypertension, diabetes, obesity, } \\
\text { hyperlipidemia, caner } \\
\text { Symptom data Physical symptoms, emotional } \\
\text { symptoms, behavioral symptoms } \\
\text { Examination data Hemoglobin gure, albumin } \\
\text { gure, AST,ALT, uric acid gure, calcium gure etc } \\
\text { Health data Blood pressure, heart pulse, } \\
\text { electrocardiogram, breathing rate, body temperature, } \\
\text { blood sugar, weight, height, physical activity, body } \\
\text { mass index }\end{array}$ \\
\hline Service data & $\begin{array}{l}\text { Bio data analysis, bio data management, exercise } \\
\text { coach services, nutrient management services }\end{array}$ \\
\hline Location data & Indoor Outdoor \\
\hline Device data & PC, IPTV, smart phone, bio signal detector \\
\hline Activity data & $\begin{array}{l}\text { Indoor temperature, outdoor temperature, indoor } \\
\text { humidity intensity of illumination, fine dust, yellow } \\
\text { dust, UV index, wind speed, amount of precipitation, } \\
\text { weather, season, time, date }\end{array}$ \\
\hline Environment data &
\end{tabular}

Table1: Context Information Classification Data [16]

The heart failure ontology [17] has developed to monitor the patients from their homes. Further, efficient knowledge representation of ontology requires some actionable parameters to calculate and trigger the specific ontology on a specific failure of patient disorders. The past history of patient disorders can be obtained with the help of biometric devices.

Knowledge representation of any domain needs some reasoning concepts with support of Decision Support System (DSS). This DSS approaches towards Artificial Intelligence (AI) system to use in real system applications to achieve objectives. The proposed system modules are shown in table II.

\begin{tabular}{|l|l|}
\hline Modules & Description \\
\hline DSS Interface & Event Occurred facilitated through DSS Interface \\
\hline DSS Control Unit & Start the intiations activities \\
\hline Factual Knowledge & Selection of relevant information from DB \\
\hline DataBase & Storage Container \\
\hline Ontology & Selected information converts into ontology language \\
\hline
\end{tabular}




\begin{tabular}{|l|l|}
\hline Rules & Applies ontology rules \\
\hline Reasoner & Conditions applied and back to ontology \\
\hline Ontology interpreter & $\begin{array}{l}\text { Ontology get responses from reasoner and give to } \\
\text { interpreter module }\end{array}$ \\
\hline Response & $\begin{array}{l}\text { At the end, response give to the user through DSS } \\
\text { interface }\end{array}$ \\
\hline
\end{tabular}

Table II. Ontology Modules [17]

The proposed architecture in [18] has used for general disease advised and provided services of knowledge centric decision support. The proposed model comprises of three main phases, (1) connected with different sources via internet to collect relevant information from heterogeneous environments, (2) Domain modelization from the sources and (3) Meta conceptualization System and operationalization exploitation. Domain modelization phase combines different knowledge domains from different sources (Domain Knowledge base). Knowledge acquisition processes may vary from the availability of standardized sources to build an efficient medical ontology. Meta conceptualization phase provides high level abstraction and designs the relationship between local and global knowledge sources. The current and future decades demands for specific medical disorders domain ontologies to treat patient on time.

A cause of death all over the world is due to chronic diseases for long term with inadequate medical treatment on time [19]. A model is proposed to efficiently control the chronic diseases with less medical costs, called ontology based Electronic Medical Record (EMR) [20]. Ontology based EMR system has captured some of the clinical vocabulary messages from HL7 RIM and also provided a less chance of errors in entry of data, availability of patient details by simultaneously multiple accesses and supports decision reasoning systems are the intentions of this proposed work.

The available chronic disease management models have used for Chronic Disease Management (CDM) and Computer based Patient Record (CPR) ontology [20]. Both models are compared with a newly proposed model and inherited some of the best features. The development cause of EMR model has due to the CDM and CPR ontologies. At the resultant, EMR ontology has mapped with HL7 RIM to insure that all the clinical information have captured.

The implementation of the BioSTROM [21] system has performed with the help of agents. Agents are the sources which collect and communicate with heterogeneous knowledge sources. Agents are based on three layer architecture of the JADE agents. Some basic steps involves in the construction of the BioSTROM system are the following.

i. Create domain ontologies

ii. Create domain methods

iii. Create configuration knowledge bases

iv. Generate JADE interfaces from the model

v. Deploy and execute

Mutual learning processes and construction of ontologies for biomedical fields are the convenient way of presentation of knowledge domains with the distributed geographically expert teams. The Clinical Practice Guideline of Diabetic Retinopathy (GLDR) [22] system has developed with guidelines of the collaborative work flows of medical experts and accessed them from distributed geographical locations.

During designing of these types of medical ontology, it requires a dedicated community to help in the identification of problems and to convert them in semi-automatic ways.

\section{B. Specific Medical Ontologies}

This section tries to cover maximum number of specific disorders medical ontologies. These medical ontologies gives description of disorders how to analyse, what are the symptoms of disorders, treatment and post treatment precautions. We attempted to explain one by one in the following ways.

- Haghighi-Koeda (HK) mood disorder [23] medical ontology has developed for mood disorder patients. This ontology is the combination of medical and psychological ontologies patterns which exchanges information between psychiatrists and psychologists to promote appropriate treatment. 
This system has enabled on web for easy access to treat patient on time and has compared with DecisionbaseTM to evaluate the results of the patients.

- Human Malaria Control Ontology (HMCO) [24] prototype has developed with the help of protégé tool and enabled on the web based access. This HMCO ontology contains 7 main parameters to get symptoms of the malaria patients to know the status of the health whether the person is under malaria attack or not. Due to these, 7 partial parameters have defined to design the malaria ontology which are: (1) Malaria vectors (2) Malaria types, (3) Malaria parasites, (4) Malaria Symptoms, (5) Malaria treatment (prevention, therapy), (6) Epidemiology data on malaria, and (7) Malaria Control [25]. Vector, Treatment, Vector Regions, Types, Parasites, Epidemiology Info, symptoms partition, Year Data, and Control [25] are the main indirect parent classes of the Human Malaria which further connect with 7 partial parameters of the human malaria.

- Mental health illness causes are still unknown and depression will be the leading disorder by year of 2020 due to unavailability of su cient medical treatment ontologies. The Mental Health Ontology [26] has proposed and comprised of three main parent classes which are disorder types, factor and treatment. Each parent class has separately explained with sub classes functionalities. This kind of mental health ontology can help to physicians, patients, researchers to efficiently to treat disorder patients on time with strengthen knowledge sources.

- Hemiparesis is the common disability issue occurred after stroke. In this disorder, mostly occurs on one side of body and sometimes leg, arm etc are damaged. Lots of researches have done to recover patient from this kind of disorder situation and most of the surveys show generic issues to stroke. So one of the well known and a specific medical ontology is formally defined known as NeuMORE [27] and it contains four phases for construction of medical ontology as follows.

Phase 1: Domain-Specific Ontology

Phase 2: Annotation of Existing Data Sources

Phase 3: RDF Database in Semantic Translation

Phase 4: Complex Query/Search in a Unified Style

OSMMI (Ontologie du Systeme Musculo-squelettique des Membres Inferieurs) [28] has

developed for the problem of lower limbs of the human body. This ontology has tried to achieve

goals of four problems related to the lower limbs such as:

1. Collect and formalize all the related materials to the problems.

2. Construct Expert System to properly diagnose the related problems.

3. On the basis of existing help, create a model for limbs problems and on the basis of this new

model, learn more from problems

4. From these steps, construct web based enabled system.

- CHRONIOUS [29] system has introduced to monitor the Chronic Obstructive Pulmonary Disease (COPD) and Chronic Kidney Disease (CKD) with support of Wearable Sensing Infrastructure (WSI). This WSI is a T-Shirt included all kinds of available sensors motes to analyse chronic diseases. These available sensors contain intelligence of medical ontologies which periodically informs doctors about the progress report of the patients.

- This paper [30] presents a framework for a family caregivers and memory loss with the support of social communities and a dedicated professional team who may accept or reject the views of the social personnel. So this framework is known as Aloa-aidants.fr [30] with support of social semiotic ontology and Aloa OIR (Open Information Retrieval) System based semiotic ontology. This semiotic ontology based system contains three views to analyse the queries of the social communities and makes the Aloa OIR system. First view is to manage and model the data in some systemic way controlled by Admin. Second, share those information managed by the Admin and giving some index to place in proper location and third point is to support bookmark functionality to the community.

- Neurological Disease (ND) ontology and NeuroPsychological Testing Ontology (NPT) [31] are considered as medical ontologies for Nervous system of the human body. The primary concern of ND ontology is on Alzheimer disease which has memory loss related disease often occurs in 40 and 50 year ages. Other related diseases in ND ontology such as dementia, multiple sclerosis, stroke and cerebrovascular disease. NPT is used for integration of various testing results for the good clinical practices. This NPT has classes and each class has subclasses. Each class or subclass of NPT 
ontology is further connected with ND ontology classes. Both ND and NPT ontologies are developed with support of OBO Foundary [32] and with some other external ontologies. ND ontology contains 400 classes, 250 textual definitions and more than 150 classes have logical definitions while in NPT ontology contains 250 specific classes.

- This paper presents Clinical based Decision Support System (CDSS) for Breast Cancer (BS) follow up with clinical practice guideline (CPG) [33]. The Breast Cancer (BC) ontology model has designed with eight classes (Patient Type, Physician Type, Illness, Menstrual Status, Recommendation, Symptom, Diagnostic Tests, Treatment, Age, Risk Factor, Weight Status and Patient Wish) with other supportive disjoined classes (Recommendation and Diagnostic Tests, and Recommendation and Treatment.) for treatment aspects and also presented in protégé tool. This breast cancer ontology model is based on rules to detect the $\mathrm{BC}$ disease.

- The MATCH CC (Colon Cancer) [34] ontology architecture system has developed and also enabled on web. In this project, the authors of OWL ontology will create the ontology of CC from existing ontologies to know the current state of the patient. The MATCH architecture occurs in two parts. In the first part, $\mathrm{CC}$ ontology has created with support of the patient profile is known as discovery phase through an ontology editor and also alongwith professionals while in second part, all the discovered information are mapped in relation database schemas.

- The ACGT MO (Advanced Clinico-Genomic Trials Medical Ontology) [35] is a medical ontology to support cancer patient treatment on time. For this purpose, they have combined clinical and molecular data with the help of Basic Formal Ontology (BFO) [36] steps to design cancer ontology from existing available ontologies such as the National Cancer Institute Thesaurus (NCIT), Gene Ontology (GO) and Foundational Model of Anatomy (FMA). These four steps of BFO are: Realism, Perspectivalism, Fallibilism and Adequatism. The Ontology-based Trial Management System (ObTiMA) has used to make sure that patient cancer treatment results are correct or not.

- Existing datasets ontologies has used to develop a new algorithm for breast cancer patients without human interactions. For this purpose, the Wisconsin Breast Cancer Dataset (WBC) [37] has used as a case study to create a new ontology engine which contains two phases. In phase 1, this algorithm will match the searched knowledge and will prepare the rules whether searched knowledge is available in the datasets or not. If this knowledge is not available in datasets, then it will be added to use in future. Phase 2 contains ontology development algorithm engine rules and these rules define concepts. This concept describes a specific domain with assumption and domain properties. In this algorithm, it contains 25 programming rules such as IF THEN ELSE, FOR, DEFINE, MAPPING, END, VARIOUS MATCHING RULES, EQUAL, NOT EQUAL etc. This algorithm has used for Benign and Malignant cancer concepts with attribute values from 1 to 10 which de ne ranges from normal to abnormal situations of cancer patient.

- Case-Based Reasoning (CBR) [38] ontology classifier has used with two open source frameworks jCOLIBRI and myCBR to find whether the patient involves in the Benign cancer or in Malignant. The jCOLIBRI is available in two versions. The version 1 is related to the designer and version 2 is related to the developer. However, version 1 is selected to create CBR due to its easy use of GUI. The jCOLIBRI is based on some reasoning structure cases while myCBR is based on classes and properties and easily implemented on protégé tool. The myCBR is a best tool for developing of a new CBR based application for breast cancer classifier.

- This is the initial work and Top level (classes \& subclasses) ontology has designed in this proposed ontology is known as disease-treatment ontology [39] after studying 50 articles related to the colon cancer therapy. The proposed ontology has used five classes to design CC therapy ontology such as: Disease, Treatment, Condition, Effect and Evidence. Further, these classes are explained subclasses with different relationship shown with supportive tool of template format.

- Computer based human diagnoses and treatment techniques require a dedicated team work such as medical administrators, medical domain experts, informatics domain experts and medical research teams. Due to these, the authors of Heart Failure (HF) ontology [40] has divided heart ontology into five main super classes which are HF Concept, Patient characterises, Testing, Treatment and Patient [40]. Each parent class is further connected with sub classes except patient class has no sub class defined. In HF concept ontology includes heart failure abnormal issues, medical synonyms and other types etc. The patient characteristic includes possible signs, diagnoses and symptoms etc. Tests 
include physical tests of the body while Treatment includes medications, devices or other recommendation related to the HF.

- This paper combines existing format structures of the patient data which can help in analysis, diagnoses and treatment steps as known is synthetic measurement [41]. This system contains patient or clinical DSS (HIMS), ontology, Sensors, Patient EHR analysis, existing literature, guideline etc are the main sources to remotely monitor the heart patient [41]. These sources of information are connected with patient mobile device and clinician mobile device to get data from patient wearable sensors.

- The authors have proposed ontology framework for chest pain assessment with the help of dynamic questionnaires. This framework contains four components with two GUIs (one GUI is for patient and other for doctors to recommend further steps against chest pain) which are adaptive questionnaire, patient and family medical history, risk assessment and clinical decision support system [42]. These components have connected with various chest pain classifications guidelines units to evaluate chest pain patients. So these units are: NICE, ACC (American College of Cardiology) and SIGN (Scottish Intercollegiate Guideline Network) [42].

- This work presents respiratory disease related issues how these kinds of information can gather from scattered environment. For this purpose, authors have discussed architecture of semantic health information query [43] with various reasoning rules along with ontology concepts. So respiratory system ontology has been developed with the following classes and subclasses. The parent root node class is Respiratory diseases with connected sub classes: pneumoconiosis and other lung diseases due to external agents, Acute Respiratory infection, chronic obstructive pulmonary disease and allied conditions etc are the sub classes of the parent node class and further these sub classe are connected with other sub classes to return query results.

- Stress is one of the leading disorder problem approach towards life threatening issues such as heart attack and cancer [44]. Due to these reasons, this paper presents ontology-Based Psychiatric Disorder Detection System which is based on a decision tree algorithm with support of meridian method [44]. In this proposed architecture, ontology has divided into two types: ontology task and ontology domain, in which ontology task has mapped on ontology domain to satisfy the required results. Further, this architecture contains GUI, inference mechanism, ontology domain, ontology task, decision tree algorithm and Maridian with DSM (Diagnostic and Statistical Manual of Mental Disorders) [44].

\section{Mass Casualties Incidents (MCI) Ontologies}

Life is the precious diamond to keep save from disasters such as Earthquakes, Plane crash, Flood, Explosion, Wildfire, underground construction, Car incidents, Cave or building collapses, etc [45]. A lot of researches have been done and still are on going to introduce new mechanisms to save the humanity from disasters. Mass Casualty Incidents (MCI) is of them to save injured people lives from incidents. Today's, MCI based ontologies are trying to develop and calculate automatically number of injured people and also recognize their injury classes. Therefore, MCI needs various ontologies for right decisions on time. Due this, various kinds of MCI design can be available for health and safety as shown below.

- $\quad$ MCI General

- $\quad$ MCI Health

- MCI Operation based

- $\quad$ MCI Hybrid

MCI Rescue, MCI Telecommunication are branches of MCI operation based. So, we consider mainly two MCI's, MCI Operation support ontologies and MCI Health-oriented ontologies because these two MCI's cover all types of MCI ontologies as explained in subsections respectively.

\section{C.1 MCI Operation support ontologies}

After 9/11 incidents in the United States, all the existing security protocols have strengthen and new security protocols have developed to handle the worst case situations [46]. Therefore, MCI is one of them newly proposed security protocol to measure and handle all kinds of situation during MCI. For this purpose, the 
newly manual system is introduced with three steps to help injured people which are Triage, Treatment and Transportation system [45][46][47][50] assigns responsibilities to each helping group during MCI.

Triage is used first arrival of emergency and medical sta in incident location and each victim person is given one minute to check health status and assign particular color tag according to injury level. In North America, START system (Simple Triage and Rapid Treatment) [45] has introduced and considered the easiest way to find victim people with three necessary parameters such as breathing, circulation and consciousness [45]. Regarding these three parameters, There are four color tags used to represent treatment level of victim persons as explained below.

Green color tag person is not a seriously injured and can get out from incident place to go hospital.

Yellow color tag person is a injured and cannot get out from incident place by its own walking.

Red color tag person is a very serious injured, cannot walk and life is in threatening situation person who needs special care of all the colors.

Black color tag person is a deceased person.

The purpose of these color tags have used due to limited number of medical and emergency staff and also limited time to save lives of victim people at incident locations.

Treatment is the second step after sorting out color tags to each injured person. The treatment locations will be made on walking distance from incident location and must assign colors to each room / units to represent patients care units. Green color and Yellow color tags patients can treat in nearby treatment units while red color tag patients must be transported on need to the hospitals and same is followed by yellow color tagged patients and Green tagged patients if they really need hospital. There must be separate location for deceased persons near to incident location not to interrupt flow of work for patients.

Transport is the final step after successful completion of a Triage and Treatment steps. In this transportation step, Priority for transportation will be given to the serious injured persons with color tags red, yellow and green respectively. Personal cars, Fire fighter trucks, police cars and helicopters etc can be used for priority based to move injured and ill people to the hospitals.

Allocation of medical staff, availability of routes for ambulances during mass casualties, allocation of hospitals for specific injured people etc, all these have done manually with cooperation of emergency staff. Due to this manual cooperation, it requires more time which cannot be tailored in real time critical situations.

All exercises have been done in manual system which comprises of Triage, Treatment and Transportation steps and also suggested automation in this existing system as shown in Fig 3 [45]. All types of resources allocation to victim people are performed by emergency and medical staff. Due to limited time and limited number of staff available during mass casualties to check the status of victim people requires a efficient solution to calculate number of available resources, allocation of hospitals to injured people are the challenging issues. For this purpose, A Spatial Decision-Support System (SDSS) has developed as shown in Fig 4 [45]. This Spatial Decision-Support System (SDSS) is a web based application and also enables the system to use anywhere with support of internet connection to update the incident locations. The SDSS system provides information of hospitals, available treatment units in hospitals, number of available beds and estimated driving distance from incident locations to surrounding hospitals with the support of centralized database.

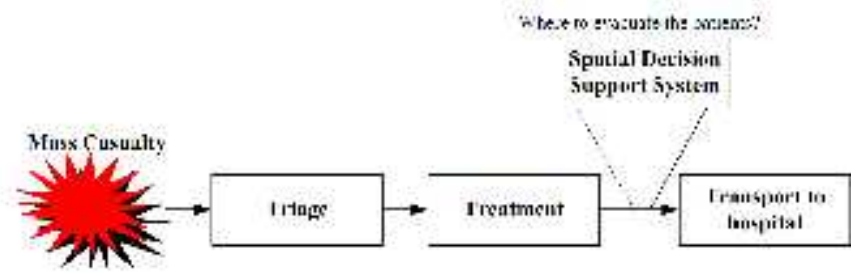

Fig. 3. An illustration shows where the suggested SDSS will fit within the pre-hospitalstage of mass casualty [45] 
This Fig 4 [45] shows two MCI's, MCI 1 and MCI 2 have occurred at the same time. Both MCI 1 and MCI 2 locations are updated through SDSS web based system on centralized database. This centralized database system calculates the paths of hospital 1 and hospital 2 from incident locations and propagates the updated information to each MCI.

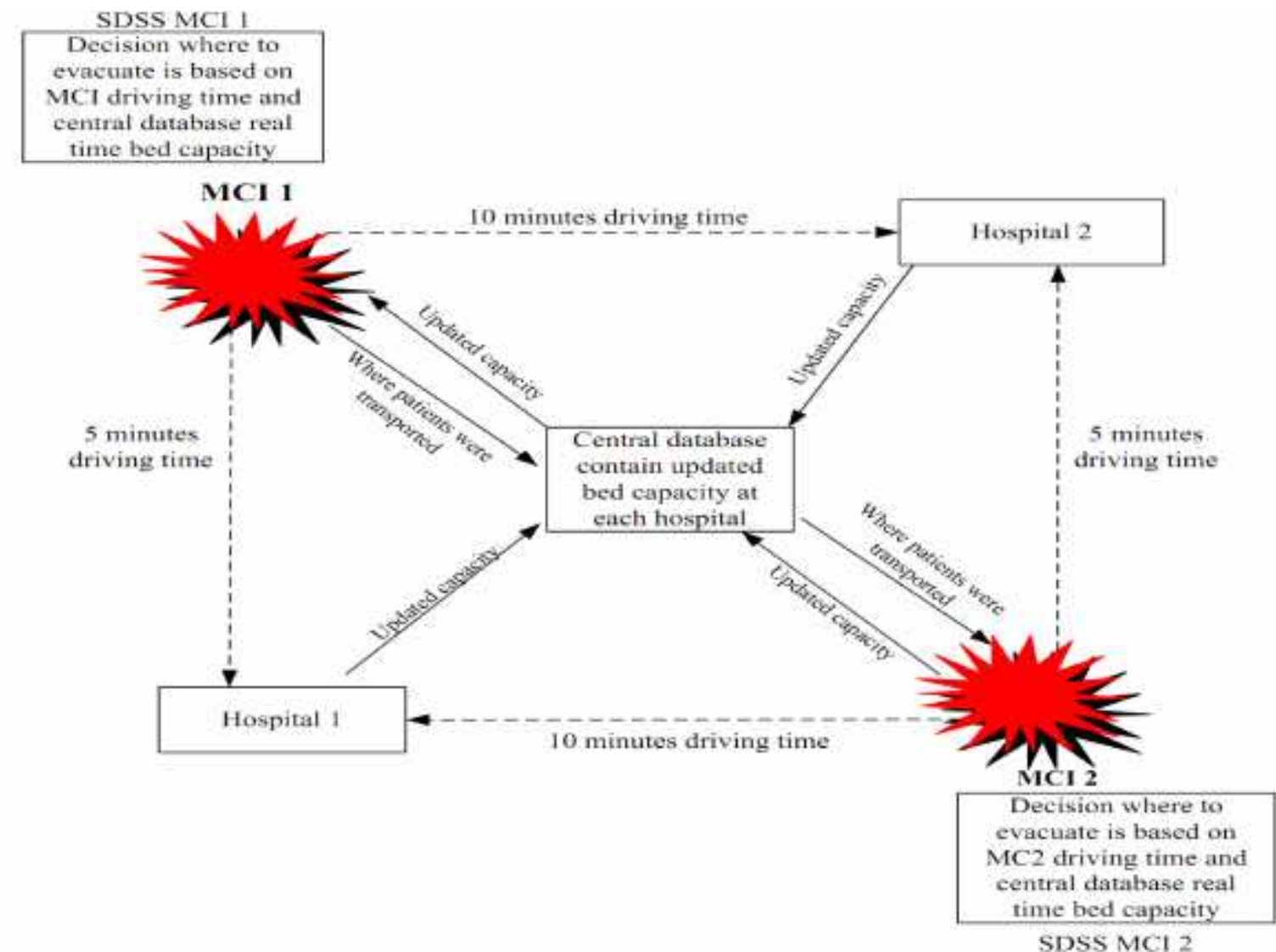

Fig. 4. An illustration shows the data flow among MCI locations and hospitals [45]

Assigning color tags, recognition of the injured people class, provide treatment at incident location, provide transportation with exact availability of treatment in hospitals etc issues faced during mass casualties incidents locations have discussed in a SDSS system. The Same issues are also raised by currently on going project SpeedUp [48]. This Speedup project focuses on two important issues that are SpeedUp Practice and SpeedUp Technology. The focal point of SpeedUp Practice is to design interactive communication model for emergency staff to give a complete structure of the mass casualty incident locations while SpeedUp Technology provides IT based solutions for emergency staff to sense the availability of the human beings in incident locations to save their lives through sensors. This kind of sensors need medical ontologies to sense the victim people and assign treatment priority to them is the motive of this SpeedUp project.

\section{C.2 MCI Health-oriented ontologies}

Mass Casualties Incidents (MCI) can occur anywhere. A lot of potentials are required to prepare tools for automatic assigning of resources (Hospitals, ambulances, emergency staff, pre calculated routes to the hospital from incident locations etc.) to victim people of the incident locations. Some of the MCIs service providers are providing both Technology and Health resources at incident locations [46][47][49][50], however, the leading underprocess and underconcerned provider of services is a SpeedUp project [48]. A 
Health oriented MCI is provided by MCI Building Community [49] and its aims to promote health and safety with cooperation of the following bodies as shown below [49].

- Pharmaceutical Companies

- Medical Device Companies

- Life Sciences Companies

- Medical Professional Societies

- International Federations

- Regulatory Bodies

More than 300 definitions of medical ontologies are covered for emergency and medical staff, victim people and vehicles etc [48] based on observations. All these information contains more than 300 concepts, 50 relations and 70 individuals implemented in protégé tool.

5. Critical Review and Future Research Directions. In the section 4 "Survey of the Existing Health Ontologies", we have studied all the existing ontologies methods related to the medical disorder analysis, diagnoses, treatment and post treatment steps. In this critical review section, we will critically analyse all the existing methods of medical ontologies and will divide into generic medical ontologies, specific medical ontologies and MCI based medical ontologies. Generic medical ontologies show how to plan and design the rules to make an efficient medical ontologies for disorder patients. All the existing work are supportive terms used for designing of medical knowledge ontologies.

All the available rules and methods in generic medical ontologies have provided repetitive structure. All these rules must study with support of domain experts and build a unique and standardized generic medical ontology which except everywhere.

Specific medical ontologies designing steps have inherited from the generic medical ontologies because all the generic medical ontologies have shown steps and techniques how to design and get data from patient body to know the disorder level. Specific medical ontologies designing have tested with various softwares and multiple specific medical ontologies rules are available for the same disorder of victim people such as Breast Cancer has presented in [33] and [37].

There should be standardized organization to check the developed specific medical ontology for which it has been designed. After successful outcome, that ontology should be implemented on all medical institutions.

MCI provides two MCIs; operation support ontologies and MCI Health-Oriented ontologies. The operation support ontologies are based on the communication infrastructure. While MCI Health-Oriented ontologies are based to design a real time system. This ontology must provide the following services as shown below. . Recognise the victim people

- Make classes for each injury and assign those injured people to a particular class

- Find the nearest hospital to provide the required treatment for injured people etc are the challenging tasks.

In current situation, there is no implemented system available to provide services to victim people of mass casualties incidents locations. The current and future decades demand to develop an automation approach in the technology to monitor the victim people of the MCI locations and provide treatment services where the victim people need it. However, this automation approach can build with support of health oriented ontologies and operation support ontologies.

\section{Conclusion}

In this paper, we have analyzed the existing literature reviews in the capacity of three main medical ontologies to help the patients on time everywhere with enabled web access. So these medical ontologies are: generic medical ontologies, specific medical ontologies and MCI based ontologies.

The generic medical ontologies show generic knowledge to help us in analysis, design and implementation of the general medical ontologies. The specific medical ontologies is the extended version of the generic medical ontologies to help in designing of a specific disorder medical ontology because all the analysis results are taken from generic medical ontology. Mass Casualties Incidents (MCIs) can be happened everywhere, to collect the data of victim people at the incident location, make the classes for each type of injured people and 
assign the injured people to the particular class and provides medical staff, ambulances, shortest routines to the hospitals, number of available beds, operation theatres etc are the leading issues faced during MCIs. Generic and Specific medical ontologies give directions how to design and implement patient disorder ontologies but the issue is how to calculate and arrange these resources during MCI victim people is the challenging task.

The current decade demands to provide health facilities with support of IT infra structure.

\section{REFERENCES}

[1] Gerkey, B., Vaughan, R. T., \& Howard, A. (2003).The player/stage project: Tools for multi-robot and distributed sensor systems. In Proceedings of the 11th international conference on advanced robotics (Vol. 1, pp. 317-323)..

[2] Koren, Y., Heisel, U., Jovane, F., Moriwaki, T., Pritschow, G., Ulsoy, G., \& Van Brussel, H. (1999). Reconfigurable manufacturing systems. CIRP Annals-Manufacturing Technology, 48(P, 527-540.

[3] Åström, K. J., \& Wittenmark, B. (2011). Computer-controlled systems: theory and design. Courier Dover Publications.

[4] http://www.who.int/mediacentre/factsheets/fs310/en/ Accessed on:28th Dec, 2012.

[5] http://en.wikipedia.org/wiki/Incidents during the Hajj Steve Roessingh, Idical Ontology Views", 2009.

[6] Subhashini, R., \& Akilandeswari, J. (2011). A survey on ontology construction methodologies. International Journal of Enterprise Computing and Business Systems, 1(1), 60-72.

[7] Wache, H., Voegele, T., Visser, U., Stuckenschmidt, H., Schuster, G., Neumann, H., \& Hübner, S. (2001, August). Ontology-based integration of information-a survey of existing approaches. In IJCAI-01 workshop: ontologies and information sharing (Vol. 2001, pp. 108-117).

[8] Maizatul A, Ismail, MY \& Sameem AK, (2006) Ontology Construction: An Overview", Malaysia

[9] Fernández-López, M., \& Gómez-Pérez, A. (2002). Overview and analysis of methodologies for building ontologies. The Knowledge Engineering Review, 17(02), 129-156.

[10] Nelson Leung, S.K Lau and J. Fan , "Enhancing the Reusability of Inter-organizational knowledge: an ontology based collaborative knowledge management Network", Electronic journal of knowledge management,ISSN 14794411, Vol 7, Issue 2,PP.233-244. www.ejkm com.

[11] Leung, N. K., Lau, S. K., \& Tsang, N. (2013). An ontology-based collaborative inter-organisational knowledge management network (CIK-NET). Journal of Information \&amp; Knowledge Management, 12(01).

[12] Tokosumi, A., Matsumoto, N., \& Murai, H. (2007, May). Medical ontologies as a knowledge repository. In Complex Medical Engineering, 2007. CME 2007. IEEE/ICME International Conference on (pp. 487-490). IEEE.

[13] Tokosumi, A., Matsumoto, N., \& Murai, H. (2007, May). Medical ontologies as a knowledge repository. In Complex Medical Engineering, 2007. CME 2007. IEEE/ICME International Conference on (pp. 487-490). IEEE.

[14] Yoshikawa, S., Satou, K., \& Konagaya, A. (2004). Drug interaction ontology (DIO) for inferences of possible drug-drug interactions. Medinfo, 11, 454-458

[15] Hadzic, M., \& Chang, E. (2005, January). Ontology-based support for human disease study. In System Sciences, 2005. HICSS'05. Proceedings of the 38th Annual Hawaii International Conference on (pp. 143a-143a). IEEE

[16] Dillon, T. S., Chang, E., \& Hadzic, M. (2008, June). Ontology support for biomedical information resources. In Computer-Based Medical Systems, 2008. CBMS'08. 21st IEEE International Symposium on (pp. 7-16). IEEE

[17] Hadzic, M., \& Chang, E. (2009). Web semantics for intelligent and dynamic information retrieval illustrated within the mental health domain. In Advances in Web Semantics I (pp. 260-275). Springer Berlin Heidelberg.

[18] Ryu, J. K., Kim, J. H., Chung, K. Y., Rim, K. W., \& Lee, J. H. (2011, April). Ontology based context information model for u-Healthcare Service. In Information Science and Applications (ICISA), 2011 International Conference on (pp. 1-6). IEEE.

[19] Jovic, A., Prcela, M., \& Gamberger, D. (2007, June). Ontologies in medical knowledge representation. In Information Technology Interfaces, 2007. ITI 2007. 29th International Conference on (pp. 535-540). IEEE. 
[20] Djedidi, R., \& Aufaure, M. (2007, June). Medical domain ontology construction approach: a basis for medical decision support. In Computer-Based Medical Systems, 2007. CBMS'07. Twentieth IEEE International Symposium on (pp. 509-511). IEEE.

[21] World health organization (WHO), "chronic diseases",http://www.who.int/topics/chronic diseases/en/ Retrieved: May 25, 2012.

[22] Iqbal, A. M., Shepherd, M., \& Abidi, S. S. R. (2011, January). An ontology-based electronic medical record for chronic disease management. In System Sciences (HICSS) 2011 44th Hawaii International Conference on (pp. 1-10). IEEE.

[23] Nyulas, C. I., O'Connor, M. J., Tu, S. W., Buckeridge, D. L., Okhmatovskaia, A., \& Musen, M. A. (2008, December). An ontology-driven framework for deploying jade agent systems. In Web Intelligence and Intelligent Agent Technology, 2008. WI-IAT'08. IEEE/WIC/ACM International Conference on (Vol. 2, pp. 573-577). IEEE

[24] Gacitua, R., Arguello Casteleiro, M., Sawyer, P., Des, J., Perez, R., Fernandez-Prieto, M. J., \& Paniagua, H. (2009, April). A collaborative workflow for building ontologies: A case study in the biomedical field. In Research Challenges in Information Science, 2009. RCIS 2009. Third International Conference on (pp. 121-128). IEEE.

[25] Haghighi, M., Koeda, M., Takai, T., \& Tanaka, H. (2009). Development of clinical ontology for mood disorder with combination of psychomedical information. J Med Dent Sci, 56(1), 1-15

[26] Daramola, O., \& Fatumo, S. (2009). Developing Ontology Support for Human Malaria Control Initiatives. ICBO, 59.

[27] Snow, R. W., Guerra, C. A., Noor, A. M., Myint, H. Y., \& Hay, S. I. (2005). The global distribution of clinical episodes of Plasmodium falciparum malaria. Nature, 434(7030), 214-217.

[28] Hadzic, M., Chen, M., \& Dillon, T. S. (2008, November). Towards the mental health ontology. In Bioinformatics and Biomedicine, 2008. BIBM'08. IEEE International Conference on (pp. 284-288). IEEE.

[29] Townsend, C., Huang, J., Dou, D., Liu, H., He, L., Hayes, P., ... \& Liu, W. (2010, December). NeuMORE: Ontology in stroke recovery. In Bioinformatics and Biomedicine Workshops (BIBMW), 2010 IEEE International Conference on (pp. 821-822). IEEE.

[30] Dao, T. T., Marin, F., \& Tho, M. H. B. (2007, August). Ontology of the musculo-skeletal system of the lower limbs. In Engineering in Medicine and Biology Society, 2007. EMBS 2007. 29th Annual International Conference of the IEEE (pp. 386-389). IEEE.

[31] Rosso, R., Munaro, G., Salvetti, O., Colantonio, S., \& Ciancitto, F. (2010, August). CHRONIOUS: an open, ubiquitous and adaptive chronic disease management platform for chronic obstructive pulmonary disease (COPD), chronic kidney disease (CKD) and renal insufficiency. In Engineering in Medicine and Biology Society (EMBC), 2010 Annual International Conference of the IEEE (pp. 6850-6853). IEEE.

[32] Tixier, M., \& Lewkowicz, M. (2011, May). Supporting the knowledge sharing dimension of social support: The case of Aloa-aidants. fr. In Collaboration Technologies and Systems (CTS), 2011 International Conference on (pp. 249-256). IEEE.

[33] Cox, A. P., Jensen, M., Duncan, W., Weinstock-Guttman, B., Szigeti, K., Smith, B., \& Diehl, A. D. (2012). Ontologies for the study of neurological disease. ICBO, 2012, 3rd

[34] Smith, B., Ashburner, M., Rosse, C., Bard, J., Bug, W., Ceusters, W., ... \& Lewis, S. (2007). The OBO Foundry: coordinated evolution of ontologies to support biomedical data integration. Nature biotechnology, 25(11), 1251-1255.

[35] Abidi, S. R. (2007, June). Ontology-based modeling of breast cancer follow-up clinical practice guideline for providing clinical decision support. In Computer-Based Medical Systems, 2007. CBMS'07. Twentieth IEEE International Symposium on (pp. 542-547). IEEE.

[36] Siddiqi, J., Akhgar, B., Gruzdz, A., Zaefarian, G., \& Ihnatowicz, A. (2008, April). Automated diagnosis system to support colon cancer treatment: MATCH. In Information Technology: New Generations, 2008. ITNG 2008. Fifth International Conference on (pp. 201-205). IEEE.

[37] Brochhausen, M., Weiler, G., Cocos, C., Stenzhorn, H., Graf, N., Dorr, M., \& Tsiknakis, M. (2008, June). The ACGT Master Ontology on Cancer-a New Terminology Source for Oncological Practice. In Computer-Based Medical Systems, 2008. CBMS'08. 21st IEEE International Symposium on (pp. 324-329). IEEE

[38] Kharbat, F., \& Ghalayini, H. (2009, April). New Algorithm for Building Ontology from Existing Rules: A Case Study. In Information Management and Engineering, 2009. ICIME'09. International Conference on (pp. 12-16). IEEE

[39] Lotfy Abdrabou, E. A. M., \& Salem, A. (2010, October). A breast cancer classifier based on a combination of case-based reasoning and ontology approach. In Computer Science and Information Technology (IMCSIT), Proceedings of the 2010 International Multiconference on (pp. 3-10). IEEE. 
[40] Khoo, C. S., Na, J. C., Wang, V. W., \& Chan, S. (2011). Developing an ontology for encoding disease treatment information in medical abstracts. DESIDOC Journal of Library \& Information Technology, 31(2).

[41] Jovic, A., Gamberger, D., \& Krstacic, G. (2011). Heart failure ontology. Bio-Algorithms Med-Syst, 7, 101-110..

[42] Fortier, P. J. (2010, July). Derivation of Non-Intrusive Cardiac Synthetic Sensor Using Native Instrumentation Metadata. In Sensor Technologies and Applications (SENSORCOMM), 2010 Fourth International Conference on (pp. 95-100). IEEE.

[43] Farooq, K., Hussain, A., Leslie, S., Eckl, C., \& Slack, W. (2011, May). Ontology-driven cardiovascular decision support system. In Pervasive Computing Technologies for Healthcare (PervasiveHealth), 2011 5th International Conference on (pp. 283-286). IEEE.

[44] Gao, S., Anton, F., Mioc, D., \& Boley, H. (2012). Non-Spatial and Geospatial Semantic Query of Health Information. International Archives of the Photogrammetry, Remote Sensing and Spatial Information Sciences, 39, B2.

[45] Lin, Y. C. (2010). Design and implementation of an ontology-based psychiatric disorder detection system. WSEAS Transactions on Information Sciences and Applications, 7(1), 56-69.

[46] http://en.wikipedia.org/wiki/Mass-casualty incident, Accessed date.10th Nov,2012.

[47] Amram, O. (2011). Emergency Care-A Web-Based Spatial System Assists Patient Evacuation during a Mass Casualty. GeoWorld, 24(10), 22..

[48] http://www.geoplace.com/ME2/dirmod.asp?sid=\&nm=\&type=MultiPublishing\&mod=PublishingTitl es\&mid=13B2F0D0AFA04476A2ACC02ED28A405F\&tier=4\&id=4569304D81BE45AB9097176F B3758A8B, Accessed date.11th Nov,2012

[49] http://www.speedupprojekt.de/en/SpeedUppagep9.html

AND http://users.minet.unijena.de/ ukrueger/mci-ontology/index-en.html, Accessed Date: 11th Nov, 2012.

[50] http://www.mcigroup.com /media/MCI/Files/IndustryPracticeInserts/Industry Practice Healthcare Final.ashx. Accessed on: 13 $3^{\text {th }}$ Nov, 2012.

[51] "ESTABLISHING A MASS CASUALTY MANAGEMENT $\quad$ SYSTEM" http://www.paho.org/english/ped/masscas.pdf, Accessed Date:13th Nov, 2012. 